\title{
Salutogenesis: Sense of Coherence in Adolescence
}

\author{
Orna Braun-Lewensohn, Orly Idan, Bengt Lindström, \\ and Malka Margalit
}

\section{Introduction}

The adolescent, at the very best, can only have gained a tentative strong SOC, which may be useful for a short-range prediction about coping with stressors and health status (Antonovsky, 1987, p. 107).

Adolescence is a period of growth and development between childhood and adulthood. This developmental period involves new demands on the individual. A major task of this period is moving toward independence from dependency on the family; therefore, peers become a crucial socialization circle for the adolescent (Romeo, 2013; Spear, 2013). During this period, a number of physiological and cognitive changes occur while individuals are confronted with developmental tasks and challenges. During the last decade, there has been a marked increase in neurobiological research on the cognitive, emotional, and behavioral changes as well as development that occur during adolescence. These studies have found that cognitively, the adolescents as the adults are capable to suppress responses when no emotional information is provided (Tottenham, Hare, \& Casey, 2011). However, it is the avoidance of social

O. Braun-Lewensohn $(\bowtie)$

Conflict Management and Resolution Program, Ben-Gurion University of the Negev, Beer Sheva, Israel

e-mail: ornabl@bgu.ac.il

O. Idan

Psychology of Intergroup Conflict and Reconciliation Lab, School of Psychology, Interdisciplinary Center (IDC), Herzliya, Israel e-mail: idan.orly08@gmail.com

B. Lindström

NTNU Center for Health Promotion and Resources, Norwegian University of Science and Technology, Trondheim, Norway e-mail: bengtblind@hotmail.com

M. Margalit

Peres Academic Center, Tel Aviv University, Rehovot, Israel

e-mail: malka@post.tau.ac.il cues during challenging situations in which adolescents have a difficulty to make a proper and rational response (Casey \& Caudle, 2013). Thus, it seems that tension between regulation of behavior and sensitivity to positive environmental cues makes the response of the individual during the period of adolescence more complex (Somerville, Hare, \& Casey, 2011).

In line with the positive youth development perspectives (Damon, 2004), there is a growing recognition of the individuals who are eager to explore the world, to acquire competence and to struggle with challenges and difficulties. This approach focuses on productive activities rather than on trying to cure and treat maladaptive tendencies. The agenda is to maximize the potential of the individual and by this to reduce the potential of hazardous, destructive, and antisocial behaviors (Lerner \& Benson, 2003). The period of adolescence is a particularly important developmental stage, since social, emotional, and cognitive processes are involved in the attempts to navigate the increasingly complex relationships (Blakemore \& Mills, 2014). Indeed, it is during these years that abstract thinking and cognitive processing develops along with enhanced moral reasoning and judgment. These positive processes enable the adolescent to explore the world, gain competences, and contribute to the world surrounding him/her (Damon, 2004). As children grow older, their coping repertoire expands and shifts from primarily external, behavior-oriented to more internal, cognitively based strategies (Aldwin, 1994).

The advanced forms of reflection such as the ability to consider things in hypothetical and abstract terms and the ability to monitor one's own cognitive activity during the process of thinking enable adolescents to see from the perspective of other persons, to plan ahead, to anticipate the future consequences of an action, and to offer alternative explanations of events. Cognitive mastery is therefore an important contribution to young people's ability to manage or regulate their feelings and to control their emotions and/or avoid being overwhelmed by them (Garnefski et al., 2001). 
These abilities have also the potential to influence the emotional-motivational and behavioral components of SOC. It is during these years that as young people move from one experience of using certain coping resources to another, different resources can be reviewed and crystallized.

In the following section, we present special adjustments that have been done for the SOC questionnaire along with multitudes studies that focused on this development period and took different directions in the study of salutogenesis during adolescence.

\section{SOC Studies During Adolescence}

Since there are hundreds of papers and studies regarding adolescents and SOC, for the purpose of this chapter we decided to narrow down our search and thus the amount of papers included in this review. Our search included sites such as-Ebsco, PsycInfo, PubMed, SocioFile, and GoogleScholar, and in addition we searched the sites of Sage, Springer, and Wiley. We looked at the last decade from 2003 to 2013 and included the search words: adolecs*, youth, sense of coherence, and salutogenesis. We came up with more than 60 articles and research from 18 countries including Scandinavian countries, South Africa, Europe, Middle East, Australia, and the United States. Table 14.1 summarizes these studies.

The following themes emerged from the identified studies:

\section{Adaptations of the Sense of Coherence Questionnaires}

Based on the original SOC questionnaire (Antonovsky, 1983), several researchers have examined the adaptability of the questionnaire to adolescent population. For example, the adolescent sense of coherence scale was adjusted to fit adolescents' characteristics - that is, development of selfidentity, orientation to one's self-society, confusion, unpredictable changes, close emotional ties with parents for the development of open communication, stability of the community, etc. Thus, several items were removed from the original 29 items scale and other were rephrased to make sure that the adolescents understand the items (Antonovsky \& Sagy, 1986), ending up with the final version of 13 items which has been considered a single factor and not the three separate components-meaningfulness, comprehensibility, and manageability (Hagquist \& Andrich, 2004). Since the original use of the updated scale many studies used this version and reliability proved to be very $\operatorname{good}(\alpha \approx .80)$. Another approach to the adaptation of the scale to the adolescence age stage was based on the use of the children version (CSOC) without the examples and distractors that were requested for the younger children. The description of the CSOC can be found in the chapter on children. The adolescence adaptation from the CSOC consisted of 16 items (e.g., "When I want something I'm sure I'll get it"; "When I need help there is someone around to help me"; on a five-point Likert type scale ranging from 1 (never) to 5 (always)). A Cronbach's alpha of .78 was obtained (Levi, Einav, Ziv, Raskind, \& Margalit, 2014).

\section{Sense of Coherence Construct During Adolescence}

The stability question regarding SOC accompanied this construct since the beginning of research about it. Antonovsky and Sagy (1986) argued that SOC should be strengthening during adolescence, and stabilized toward the end of this developmental period. However, studies which addressed the issue of age and the stability of SOC revealed inconsistencies (Apres et al., 2013; Ayo-Yusuf, Reddy, \& Van Den Borne, 2008; García-Moya et al., 2013; Kristnsson \& Ohlund, 2005; Moksnes, Espnes, \& Lillefjell, 2012). Indeed, Eriksson (2007) have stated that SOC is likely to vary during adolescence due to developmental changes, transitions, and challenges. While some researchers did not find differences among various age groups (Honkinen et al., 2008) and claimed the existence of SOC stability during adolescence (KroningerJungaberle, 2013), others focused on the variability between groups of adolescents between younger and older adolescents (García-Moya et al., 2013) as well as between groups with high vs. low scores of SOC. The group with lower SOC reported more variability in its SOC scores (Buddeberg-Fischer, Klaghofer, \& Schnyder, 2001).

Moreover, during periods of political violence, studies have shed light on the impacts of fragile periods and documented a drop in SOC levels during acute stress situations. However, once the acuteness is over, the SOC gains back its strengths (Braun-Lewensohn, Sagy, Sabato, \& Galili, 2013). Nevertheless, when adolescents face chronic states of stress, such as longitudinal missile attacks, the deterioration of the SOC remained stable over time (BraunLewensohn \& Sagy, 2010). 
Table 14.1 SOC Studies during adolescence

\begin{tabular}{|c|c|c|c|c|c|}
\hline Author & Year & Place & Population & Variables & Results \\
\hline 1. Apers et al. & 2013 & Belgium & $49814-18$ years longitudinal & $\begin{array}{l}\text { QoL, SOC, age, gender, } \\
\text { educational level, disease } \\
\text { complexity, prior surgery }\end{array}$ & $\begin{array}{l}\text { The higher the SOC the higher } \\
\text { the perceived health }\end{array}$ \\
\hline $\begin{array}{l}\text { 2. Ayo-Yussuf } \\
\text { et al. }\end{array}$ & 2008 & South Africa & $\begin{array}{l}8 \text { th grade, } 3 \text { waves } \\
(18 \text { months }) 970 \text { adol } \\
\text { adolescents }\end{array}$ & $\begin{array}{l}\text { SOC, smoking, gingival health, } \\
\text { socioeconomic status, age, } \\
\text { gender, tooth brushing }\end{array}$ & $\begin{array}{l}\text { Adolescent smoking and SOC } \\
\text { levels are independent } \\
\text { predictors of self-reported } \\
\text { gingivitis. Therefore, in addition } \\
\text { to plaque control, smoking } \\
\text { prevention and the teaching of } \\
\text { stress-coping skills may be } \\
\text { important interventions for } \\
\text { promoting adolescents' gingival } \\
\text { health }\end{array}$ \\
\hline $\begin{array}{l}\text { 3. Ayo-Yussuf } \\
\text { et al. }\end{array}$ & 2009 & South Africa & $\begin{array}{l}\text { 8th grade } 774 \text { baseline; of } \\
\text { those not consistently } \\
\text { brushing twice daily } 578 \text { were } \\
\text { followed after } 18 \text { months }\end{array}$ & $\begin{array}{l}\text { Sociodemographic, depression, } \\
\text { smoking, dental treatment, SOC, } \\
\text { tooth brush motivation }\end{array}$ & $\begin{array}{l}\text { SOC, living with mother, } \\
\text { smoking, being depressed } \\
\text { vulnerable were associated with } \\
\text { the transition to twice daily } \\
\text { tooth brushing }\end{array}$ \\
\hline $\begin{array}{l}\text { 4. Ayo-Yussuf } \\
\text { et al. }\end{array}$ & 2013 & South Africa & $\begin{array}{l}\text { 1st wave- } 13-15 \text { years; } 2 \text { nd } \\
\text { wave after } 6 \text { months.; } 3 \text { rd } \\
\text { wave after } 1.5 \text { years }\end{array}$ & $\begin{array}{l}\text { SOC, exposure to household } \\
\text { smoke }\end{array}$ & $\begin{array}{l}\text { Lower SOC related to use of } \\
\text { alcohol and binge drinking at } \\
\text { base line; higher SOC linked to } \\
\text { more commitment to stay smoke } \\
\text { free. SOC better predictor than } \\
\text { self-efficacy }\end{array}$ \\
\hline 5. Baker et al. & 2010 & Malaysia & 43912 and 13 years & $\begin{array}{l}\text { SOC, self-esteem, health locus } \\
\text { of control, parents' income and } \\
\text { education, oral health status }\end{array}$ & $\begin{array}{l}\text { SOC the most important } \\
\text { psychosocial factor for oral } \\
\text { health status, better health } \\
\text { perception and functioning as } \\
\text { well as quality of life }\end{array}$ \\
\hline $\begin{array}{l}\text { 6. Bauminger } \\
\text { et al. }\end{array}$ & 2008 & Israel & $19612-15$ years & $\begin{array}{l}\text { SOC, self-disclosure, intimacy, } \\
\text { attachment style }\end{array}$ & $\begin{array}{l}\text { SOC, self-disclosure and } \\
\text { attachment style predicted } \\
\text { intimacy. Self-disclosure } \\
\text { predicted intimacy especially at } \\
\text { low levels of SOC. While SOC } \\
\text { and disclosure had direct effect } \\
\text { on intimacy, avoidant and } \\
\text { anxious attachment had indirect } \\
\text { effect via SOC and disclosure }\end{array}$ \\
\hline 7. Blom et al. & 2010 & Sweden & $\begin{array}{l}66 \text { nonclinical females } \\
(15.9-17.7) 73 \text { clinical } \mathrm{f} \\
(14.5-18.4)\end{array}$ & $\begin{array}{l}\text { SOC, anxiety, depression, self- } \\
\text { assessed health, physiological } \\
\text { parameters, emotional scales }\end{array}$ & $\begin{array}{l}\text { The SOC scale did not appear to } \\
\text { be a measure of a distinct } \\
\text { salutogenic construct, but an } \\
\text { inverse measure of persistent } \\
\text { depressive symptoms and } \\
\text { generalized social anxiety } \\
\text { similar to the diagnostic criteria } \\
\text { for major depressive disorder } \\
\text { (MDD), dysthymic disorder, } \\
\text { generalized anxiety disorder } \\
\text { (GAD) or generalized social } \\
\text { anxiety disorder (SAD) } \\
\text { according to DSM-IV }\end{array}$ \\
\hline $\begin{array}{l}\text { 8. Braun- } \\
\text { Lewensohn } \\
\text { et al. } \\
\end{array}$ & 2010 & Israel & $\begin{array}{l}114(2006) ; 83(2009) 12-18 \\
\text { years }\end{array}$ & SOC, hope, values & $\begin{array}{l}\text { SOC, hope, and values } \\
\text { decreased following years of } \\
\text { political violence }\end{array}$ \\
\hline $\begin{array}{l}\text { 9. Braun- } \\
\text { Lewensohn } \\
\text { et al. }\end{array}$ & 2011 & Israel & $23012-18$ years & $\begin{array}{l}\text { Anxiety, anger, SPD, SOC, } \\
\text { exposure, demographics }\end{array}$ & $\begin{array}{l}\text { SOC mediated the relationships } \\
\text { between exposure to missile } \\
\text { attacks and stress reactions }\end{array}$ \\
\hline $\begin{array}{l}\text { 10. Braun- } \\
\text { Lewensohn } \\
\text { et al. }\end{array}$ & 2011 & Israel & $\begin{array}{l}160912-18 \text { years Jews, } \\
\text { Druze, Muslims after bush- } \\
\text { fire }\end{array}$ & $\begin{array}{l}\text { Personal and community SOC, } \\
\text { anxiety, anger, SPD }\end{array}$ & $\begin{array}{l}\text { Personal SOC stronger among } \\
\text { majority (Jews); predicted stress } \\
\text { in all groups; community SOC } \\
\text { strongest among Druze } \\
\text { predicted stress only for Druze }\end{array}$ \\
\hline
\end{tabular}


Table 14.1 (continued)

\begin{tabular}{|c|c|c|c|c|c|}
\hline Author & Year & Place & Population & Variables & Results \\
\hline $\begin{array}{l}11 . \text { Braun- } \\
\text { Lewensohn } \\
\text { et al. }\end{array}$ & 2011 & Israel & $12-18$ years & $\begin{array}{l}\text { Anxiety, anger, SPD, cognitive } \\
\text { appraisal, coping strategies, } \\
\text { SOC }\end{array}$ & $\begin{array}{l}\text { SOC was the strongest direct } \\
\text { and indirect predictor of stress. } \\
\text { Found to be important also in } \\
\text { acute stress situation }\end{array}$ \\
\hline $\begin{array}{l}\text { 12. Braun- } \\
\text { Lewensohn } \\
\text { et al. }\end{array}$ & 2011 & Israel & $\begin{array}{l}12-18 \text { years Jews-138, } \\
\text { Bedouin-Arabs- } 84 \text { during } \\
\text { missile attacks }\end{array}$ & Anxiety, anger, SPD, SOC, hope & $\begin{array}{l}\text { SOC higher among Jews; anger } \\
\text { and collective hope higher } \\
\text { among Arabs; SOC explained } \\
\text { stress among Jews; Hope among } \\
\text { Arabs }\end{array}$ \\
\hline $\begin{array}{l}\text { 13. Braun- } \\
\text { Lewensohn } \\
\text { et al. }\end{array}$ & 2013 & Israel & $\begin{array}{l}\text { 12-18 years } 104 \text { during } \\
\text { disengagement from Gaza; } \\
773 \text { months after } \\
\text { disengagement; } 1155 \text { years } \\
\text { after disengagement }\end{array}$ & $\begin{array}{l}\text { Anxiety, anger, SOC, sense of } \\
\text { community }\end{array}$ & $\begin{array}{l}\text { Levels of anxiety (but not of } \\
\text { anger) dropped after } 5 \text { years; } \\
\text { SOC decreased at } 3 \text { months but } \\
\text { recovered at } 5 \text { years. SOC was } \\
\text { the strongest predictor of stress } \\
\text { and best predicted anxiety at } \\
3 \text { months and } 5 \text { years }\end{array}$ \\
\hline $\begin{array}{l}\text { 14. Braun- } \\
\text { Lewensohn }\end{array}$ & 2013 & Israel & $\begin{array}{l}\text { 12-18 years-A year after a } \\
\text { natural disaster of bush fire } \\
\text { Jew-413, Druze-356, } \\
\text { Muslims-374 }\end{array}$ & $\begin{array}{l}\text { Personal and community SOC, } \\
\text { anxiety, anger, psychological } \\
\text { distress, demographics }\end{array}$ & $\begin{array}{l}\text { The Jewish majority had higher } \\
\text { personal SOC compared to } \\
\text { minority groups. Community } \\
\text { SOC was highest among the } \\
\text { collectivist Druze culture. } \\
\text { Personal SOC had significant } \\
\text { contribution to stress reactions } \\
\text { in all cultures. Community SOC } \\
\text { had contribution to stress only } \\
\text { among Druze }\end{array}$ \\
\hline 15. Broni-kowski & 2010 & Poland & $\begin{array}{l}38 \text { boys and } 33 \text { girls- } \\
\text { experimental group; } 34 \text { boys } \\
\text { and } 32 \text { girls control ( } 13 \text { years) }\end{array}$ & $\begin{array}{l}\text { SOC, physical fitness, body } \\
\text { constituency, frequency of } \\
\text { leisure-time physical activity }\end{array}$ & $\begin{array}{l}\text { Boys and girls from intervention } \\
\text { groups maintained high level of } \\
\text { leisure-time physical activity } \\
\text { after the program. No distinctive } \\
\text { differences were found in case } \\
\text { of body constituency between } \\
\text { experimental and control groups } \\
\text { except for muscle mass and sum } \\
\text { of skinfolds in girls. In sense of } \\
\text { coherence, gradual increase was } \\
\text { noticed from pretest to follow up } \\
\text { in experimental boys, whereas } \\
\text { in girls it was increased at } \\
\text { posttest but later at follow-up it } \\
\text { dropped. In control groups, level } \\
\text { of coherence was declining } \\
\text { during the whole duration of the } \\
\text { study. Confirm effectiveness of } \\
\text { a multilevel intervention } \\
\text { designed to increase sense of } \\
\text { coherence and promote self- } \\
\text { responsibility in health-related } \\
\text { lifestyle }\end{array}$ \\
\hline $\begin{array}{l}\text { 16. Broni-kowski } \\
\text { et al. }\end{array}$ & 2009 & Poland & $\begin{array}{l}199 \text { 13-year boys: } \\
\text { experimental } \\
\text { vs. nonexperimental group }\end{array}$ & $\begin{array}{l}\text { SOC, cardiorespiratory fitness, } \\
\text { physical activity. Intervention: } \\
\text { physical education program for } \\
\text { responsibility of physical } \\
\text { activity }\end{array}$ & $\begin{array}{l}\text { Only experimental group } \\
\text { improved SOC and } \\
\text { cardiorespiratory fitness }\end{array}$ \\
\hline 17. Dorri et al. & 2010 & Iran & 1054 6th graders & $\begin{array}{l}\text { Sociodemographic, tooth } \\
\text { brushing, SOC }\end{array}$ & $\begin{array}{l}\text { Higher SOC more tooth } \\
\text { brushing, regardless gender and } \\
\text { father's education; boys stronger } \\
\text { SOC than girls }\end{array}$ \\
\hline 18. Edbom et al. & 2010 & Sweden & 312 twins 16 and 21 years & ADHD, SOC (longitudinal) & High SOC protective of ADHD \\
\hline 19. Evans et al. & 2010 & USA & 1619 8th and 10th grade & $\begin{array}{l}\text { SOC, protection and risk factors, } \\
\text { gender }\end{array}$ & $\begin{array}{l}\text { Multiple ecological domains are } \\
\text { useful for understanding SOC }\end{array}$ \\
\hline
\end{tabular}


Table 14.1 (continued)

\begin{tabular}{|c|c|c|c|c|c|}
\hline Author & Year & Place & Population & Variables & Results \\
\hline 20. Feldt et al. & 2005 & Finland & $\begin{array}{l}2028,9,14,27,36, \text { and } \\
42 \text { years longitudinal }\end{array}$ & $\begin{array}{l}\text { Child-centered parenting, } \\
\text { parental SES, school success in } \\
\text { adolescence, education, career } \\
\text { stability, adult SOC }\end{array}$ & $\begin{array}{l}\text { Child-centered parenting, high } \\
\text { parental SES, school success at } \\
14 \text { years were indirectly } \\
\text { associated with SOC via } \\
\text { education and career stability }\end{array}$ \\
\hline 21. Fried et al. & 2010 & Israel & $106916-19$ years & $\begin{array}{l}\text { Gamboling, temperament, SOC, } \\
\text { exposure to advertising, } \\
\text { emotionality, activity, } \\
\text { sociability }\end{array}$ & $\begin{array}{l}\text { No relationships between SOC } \\
\text { and gamboling behavior or } \\
\text { problems }\end{array}$ \\
\hline $\begin{array}{l}\text { 22. García-Moya } \\
\text { et al. }\end{array}$ & 2012 & Spain & $758013-18$ years & $\begin{array}{l}\text { Family dimensions, gender, age, } \\
\text { SOC }\end{array}$ & $\begin{array}{l}\text { No gender difference on SOC; } \\
\text { younger adolescents-higher } \\
\text { SOC; family var. (easy } \\
\text { communication and parental } \\
\text { knowledge) accounted for } 18 \% \\
\text { of SOC }\end{array}$ \\
\hline $\begin{array}{l}\text { 23. García-Moya } \\
\text { et al. }\end{array}$ & 2013 & Spain & $758013-18$ years & $\begin{array}{l}\text { Health behavior, school factors, } \\
\text { SOC }\end{array}$ & $\begin{array}{l}\text { Supportive school climate and } \\
\text { SOC are relevant to adolescent } \\
\text { health; SOC most significant in } \\
\text { predicting school demands } \\
\text { stress. High SOC students } \\
\text { reported less school stress }\end{array}$ \\
\hline $\begin{array}{l}\text { 24. García-Moya } \\
\text { et al. }\end{array}$ & 2013 & Spain & $547515-18$ years & $\begin{array}{l}\text { SOC, alcohol consumption, } \\
\text { tobacco use, life time } \\
\text { drunkenness }\end{array}$ & $\begin{array}{l}\text { Higher SOC linked to reduced } \\
\text { involvement in tobacco use and } \\
\text { drunkenness. It was not } \\
\text { associated with current drinking. } \\
\text { SOC had effect on most groups } \\
\text { but not on those who consume } \\
\text { illegal drugs }\end{array}$ \\
\hline $\begin{array}{l}\text { 25. García-Moya } \\
\text { et al. }\end{array}$ & 2013 & Spain & $494313-18$ years & $\begin{array}{l}\text { SOC, parent-child relationships, } \\
\text { teacher and classmates support, } \\
\text { behavior in peer group, } \\
\text { neighborhood assets }\end{array}$ & $\begin{array}{l}\text { Parent-child relationships } \\
\text { emerged as the main contributor } \\
\text { to SOC in all sample; also the } \\
\text { other contextual factors had } \\
\text { significant contribution to SOC }\end{array}$ \\
\hline $\begin{array}{l}\text { 26. García-Moya } \\
\text { et al. }\end{array}$ & 2013 & Spain & $758013-18$ years & $\begin{array}{l}\text { SOC, neighborhood risk and } \\
\text { assets }\end{array}$ & $\begin{array}{l}\text { Neighborhood risks were } \\
\text { negatively associated with SOC; } \\
\text { neighborhood assets especially } \\
\text { relationships with significant } \\
\text { adults positively associated with } \\
\text { SOC }\end{array}$ \\
\hline 27. Gauffin et al. & 2010 & Sweden & $\begin{array}{l}97 \text { epilepsy } 13-221 \text { st wave, } \\
18-27 \text { nd wave }\end{array}$ & $\begin{array}{l}\text { Self-esteem, SOC, medical } \\
\text { condition }\end{array}$ & $\begin{array}{l}\text { SOC and self-esteem decreased } \\
\text { in the } 2 \text { nd wave; those who were } \\
\text { free of seizures had higher SOC; } \\
\text { no association between seizure } \\
\text { frequency and SOC }\end{array}$ \\
\hline 28. Geckova et al. & 2010 & Slovak & $\begin{array}{l}199214-23 \text { years- } \\
\text { secondary schools }\end{array}$ & $\begin{array}{l}\text { SES, school factors, perceived } \\
\text { social support, SOC, health }\end{array}$ & $\begin{array}{l}\text { Association between: } \\
\text { educational aspiration, parental } \\
\text { education level, father } \\
\text { unemployment, school } \\
\text { atmosphere, attitudes toward } \\
\text { school, social support from } \\
\text { father and SOC; SOC } \\
\text { contributes to reporting of } \\
\text { educational aspiration to all } \\
\text { students }\end{array}$ \\
\hline 29. Glanz et al. & 2005 & USA & 34387 th grade & Ethnicity, SOC, tobacco use & $\begin{array}{l}\text { Ethnic differences in smoking; } \\
\text { higher SOC lower risk for } \\
\text { smoking in all groups }\end{array}$ \\
\hline $\begin{array}{l}\text { 30. Gustafsson } \\
\text { et al. }\end{array}$ & 2010 & Sweden & $\begin{array}{l}15 \text { adolescents exposed to } \\
\text { child abuse }\end{array}$ & $\begin{array}{l}\text { SOC, externalizing, } \\
\text { internalizing symptoms, trauma } \\
\text { symptoms }\end{array}$ & $\begin{array}{l}\text { SOC correlated mostly to } \\
\text { externalizing and internalizing } \\
\text { symptoms but not to trauma } \\
\text { symptoms }\end{array}$ \\
\hline
\end{tabular}


Table 14.1 (continued)

\begin{tabular}{|c|c|c|c|c|c|}
\hline Author & Year & Place & Population & Variables & Results \\
\hline $\begin{array}{l}\text { 31. Hagquist } \\
\text { et al. }\end{array}$ & 2004 & Sweden & 88918 years & SOC & $\begin{array}{l}\text { The questionnaire could } \\
\text { separate the adolescents from } \\
\text { general population. The SOC } \\
\text { scale should be dealt with as a } \\
\text { measure of one global factor }\end{array}$ \\
\hline 32. Hansson et al. & 2004 & Sweden & $\begin{array}{l}186 \text { (around } 14-15 \text { at intake) } \\
\text { adolescents with conduct } \\
\text { disorder and juvenile } \\
\text { delinquency (at least } 20 \text { at } \\
\text { follow-up after intervention) }\end{array}$ & $\begin{array}{l}\text { SOC, life satisfaction, } \\
\text { symptoms checklist, antisocial } \\
\text { behavior, global functioning, } \\
\text { social functioning, social } \\
\text { belonging }\end{array}$ & $\begin{array}{l}\text { SOC is low at follow-up and } \\
\text { symptoms are higher compare to } \\
\text { regular population }\end{array}$ \\
\hline $\begin{array}{l}\text { 33. Honkinen } \\
\text { et al. }\end{array}$ & 2008 & Finland & $\begin{array}{l}84615 \text { years. } 792 \text { at follow-up } \\
18 \text { years }\end{array}$ & SOC & $\begin{array}{l}\text { Overall, there were no changes } \\
\text { in SOC scores in the entire } \\
\text { sample }\end{array}$ \\
\hline $\begin{array}{l}\text { 34. Honkinen } \\
\text { et al. }\end{array}$ & 2009 & Finland & $\begin{array}{l}12,15,18 \text { years longitudinal } \\
15 \text { years follow-up }\end{array}$ & $\begin{array}{l}\text { Psychological symptoms in } \\
\text { childhood, SOC in adolescence }\end{array}$ & $\begin{array}{l}\text { Destructive behavior at } 3 \text { years, } \\
\text { attention problems at } 12 \text { years, } \\
\text { anxiety delinquency, } \\
\text { somatization at } 15 \text { years } \\
\text { predicted poor SOC at } 18 \text { years; } \\
\text { problems reported by } \\
\text { adolescents explained better } \\
\text { poor SOC than problems } \\
\text { reported by parents }\end{array}$ \\
\hline $\begin{array}{l}\text { 35. Honkinen } \\
\text { et al. }\end{array}$ & 2005 & Finland & 123112 years & $\begin{array}{l}\text { Health behavior, SOC, school } \\
\text { marks }\end{array}$ & $\begin{array}{l}\text { Physical exercise most strongly } \\
\text { associated with health; SOC and } \\
\text { social support also associated } \\
\text { with health }\end{array}$ \\
\hline 36. Idan et al. & 2013 & Israel & $\begin{array}{l}856 \text { 10th-12th grade } \\
529 \text { achieving students; } \\
327 \text { LD students }\end{array}$ & $\begin{array}{l}\text { SOC, psychological needs, } \\
\text { loneliness, family climate, hope, } \\
\text { academic and self-efficacy, } \\
\text { effort, grades }\end{array}$ & $\begin{array}{l}\text { SOC positively correlated: } \\
\text { competence/autonomy, } \\
\text { relatedness, self-efficacy, family } \\
\text { cohesion, hope, effort. } \\
\text { Negatively correlated: } \\
\text { loneliness. SOC contributed to } \\
\text { psychological processes of all } \\
\text { students }\end{array}$ \\
\hline 37. Jaakkola et al. & 2013 & Finland & 77718 years old & $\begin{array}{l}\text { SOC, dental fear-anxiety, } \\
\text { gender, education }\end{array}$ & $\begin{array}{l}\text { High fear lower SOC also when } \\
\text { controlling for gender and } \\
\text { education }\end{array}$ \\
\hline $\begin{array}{l}\text { 38. Koushede } \\
\text { et al. }\end{array}$ & 2009 & Denmark & 13937 th-9th grade & $\begin{array}{l}\text { Demographics, medicine use, } \\
\text { psychosocial aspects, SOC }\end{array}$ & $\begin{array}{l}\text { More medicine use linked to } \\
\text { lower SOC; frequency of } \\
\text { headaches modifies association } \\
\text { between SOC and medicine use }\end{array}$ \\
\hline $\begin{array}{l}\text { 39. Koposov } \\
\text { et al. }\end{array}$ & 2003 & Russia & $\begin{array}{l}\text { 14-19 years court ordered } \\
\text { juvenile detention center }\end{array}$ & $\begin{array}{l}\text { Exposure to community } \\
\text { violence, SOC, psycho } \\
\text { pathology, problem behavior }\end{array}$ & $\begin{array}{l}\text { SOC partially mediated } \\
\text { victimization and } \\
\text { psychopathology. Higher SOC } \\
\text { potentially reduced } \\
\text { psychopathology }\end{array}$ \\
\hline $\begin{array}{l}\text { 40. Kris-tensson } \\
\text { et al. }\end{array}$ & 2005 & Sweden & $25316-21$ years & $\begin{array}{l}\text { SOC, coping resources, } \\
\text { aggression teacher evaluation, } \\
\text { school marks, gender, age }\end{array}$ & $\begin{array}{l}\text { SOC and coping resources } \\
\text { positively correlated with school } \\
\text { marks; aggression negatively } \\
\text { correlated with school marks; } \\
\text { females higher on coping } \\
\text { resources but lower on SOC and } \\
\text { aggression }\end{array}$ \\
\hline $\begin{array}{l}\text { 41. Kroninger- } \\
\text { Jungaberle } \\
\text { et al. }\end{array}$ & 2013 & Germany & $\begin{array}{l}15516 \text { years and } 19 \text { years } \\
\text { (longitudinal) }\end{array}$ & $\begin{array}{l}\text { SOC, self-efficacy, mental } \\
\text { health symptoms }\end{array}$ & $\begin{array}{l}\text { SOC and self-efficacy predicted } \\
\text { negative symptoms at } 16 \text { years } \\
\text { and at } 19 \text { years. Resilience and } \\
\text { symptoms at time } 1 \text { predicted } \\
\text { resilience and symptoms at time } \\
2 . \text { SOC at age } 16 \text { predicted } \\
\text { mental health at } 19 \text {. SOC is } \\
\text { already stable at adolescence }\end{array}$ \\
\hline
\end{tabular}


Table 14.1 (continued)

\begin{tabular}{|c|c|c|c|c|c|}
\hline Author & Year & Place & Population & Variables & Results \\
\hline 42. Lackaye et al. & 2006 & Israel & $\begin{array}{l}5717 \text { th grade with and } \\
\text { without LD }\end{array}$ & $\begin{array}{l}\text { Grades from school records, } \\
\text { gender, age, leisure activity, } \\
\text { satisfaction from these } \\
\text { activities, academic help school } \\
\text { or private, diagnosed LD, effort, } \\
\text { academic self-efficacy, } \\
\text { loneness, mood, SOC, hope }\end{array}$ & $\begin{array}{l}\text { Achievement, academic self- } \\
\text { efficacy, negative mood, hope } \\
\text { and SOC predicted effort } \\
\text { investment for students with } \\
\text { LD. The importance of SOC and } \\
\text { hope in this context is } \\
\text { highlighted }\end{array}$ \\
\hline 43. Levi et al. & 2013 & Israel & 289 10th grade students & $\begin{array}{l}\text { SOC, hope, academic } \\
\text { expectations, grades, self- } \\
\text { efficacy }\end{array}$ & $\begin{array}{l}\text { SOC and emotional self-efficacy } \\
\text { contributed to hope which in } \\
\text { turn had a significant effect on } \\
\text { grade expectation that predict } \\
\text { academic achievement }\end{array}$ \\
\hline 44. Luyckx et al. & 2012 & Belgium & $\begin{array}{l}38014-18 \text { years (other older } \\
\text { groups) }\end{array}$ & SOC, heart disease & $\begin{array}{l}\text { Adolescents' SOC lower than } \\
\text { young employed adults; heat } \\
\text { disease correlated higher SOC }\end{array}$ \\
\hline 45. Mattila et al. & 2011 & Finland & 15 years & $\begin{array}{l}\text { SOC, QoL, health behavior, } \\
\text { social competence }\end{array}$ & $\begin{array}{l}\text { Higher SOC related to lighter } \\
\text { use of alcohol, no smokers, } \\
\text { better oral care, and better social } \\
\text { competence }\end{array}$ \\
\hline 46. Modin et al. & 2011 & Sweden & 7930 9th graders & $\begin{array}{l}\text { Subjective health, school } \\
\text { working conditions, school SOC }\end{array}$ & $\begin{array}{l}\text { High levels of control and a } \\
\text { strong school-related sense of } \\
\text { coherence can protect against } \\
\text { the more detrimental effects on } \\
\text { health that high demands at } \\
\text { school may cause }\end{array}$ \\
\hline 47. Marsh et al. & 2007 & USA & 1619 middle school & SOC, risk and protective factors & $\begin{array}{l}\text { Social support, anger } \\
\text { expression, family conflict, } \\
\text { neighborhood cohesion all were } \\
\text { predictors of SOC; gang } \\
\text { membership predictor of SOC } \\
\text { for boys; age predictor of SOC } \\
\text { for girls }\end{array}$ \\
\hline 48. Myrin et al. & 2008 & Sweden & $38314-15$ years & SOC, psychosocial factors & $\begin{array}{l}\text { Girls negative outcomes on } \\
\text { psychosocial factors: } \\
\text { depression, life satisfaction, } \\
\text { worries about family member, } \\
\text { poor psychosomatic health; all } \\
\text { these related to low SOC }\end{array}$ \\
\hline 49. Myrin et al. & 2006 & Sweden & $38314-15$ years & $\begin{array}{l}\text { Socioeconomic, SOC, health } \\
\text { behavior-tobacco use, alcohol } \\
\text { consumption, eating habits }\end{array}$ & $\begin{array}{l}\text { SOC lower among girls; Low } \\
\text { SOC girls have more health } \\
\text { behavior problems; high SES } \\
\text { with low SOC adolescents } \\
\text { related to }\end{array}$ \\
\hline $\begin{array}{l}\text { 50. Moksnes } \\
\text { et al. }\end{array}$ & 2013 & Norway & $118313-18$ years & $\begin{array}{l}\text { Stress, SOC, emotional } \\
\text { symptoms }\end{array}$ & $\begin{array}{l}\text { Girls higher on: stress, peer } \\
\text { pressure, home life, school } \\
\text { performance, school leisure, } \\
\text { conflict, emotional symptoms; } \\
\text { boys higher on SOC; SOC } \\
\text { moderated the association } \\
\text { between stress-related peer } \\
\text { pressure and depression } \\
\text { symptoms for both genders }\end{array}$ \\
\hline $\begin{array}{l}\text { 51. Moksnes } \\
\text { et al. }\end{array}$ & 2011 & Norway & $118313-18$ years & $\begin{array}{l}\text { Stress, subjective health } \\
\text { complaints, SOC, peer pressure, } \\
\text { home life, school attendance }\end{array}$ & $\begin{array}{l}\text { Girls higher stress than boys; } \\
\text { boys higher SOC than girls; } \\
\text { SOC inversely related to health } \\
\text { complaints and stress; peer } \\
\text { pressure, home life, and school } \\
\text { attendance higher stress. No } \\
\text { moderation of SOC }\end{array}$ \\
\hline $\begin{array}{l}\text { 52. Moksnes } \\
\text { et al. }\end{array}$ & 2012 & Norway & $120913-18$ years & $\begin{array}{l}\text { Depression, anxiety, SOC, } \\
\text { gender, age }\end{array}$ & $\begin{array}{l}\text { Girls more anxious and } \\
\text { depressed; boys higher SOC; } \\
\text { Higher SOC less anxiety and } \\
\text { depression-the association is } \\
\text { stronger for girls }\end{array}$ \\
\hline
\end{tabular}


Table 14.1 (continued)

\begin{tabular}{|c|c|c|c|c|c|}
\hline Author & Year & Place & Population & Variables & Results \\
\hline 53. Nilsson et al. & 2007 & Sweden & 430516 and 19 years & $\begin{array}{l}\text { SOC, alcohol-related behavior } \\
\text { problems, alcohol intoxication, } \\
\text { alcohol consumption }\end{array}$ & $\begin{array}{l}\text { SOC and alcohol intoxication } \\
\text { were related to alcohol } \\
\text { problems. SOC despite } \\
\text { intoxication protect from } \\
\text { alcohol-related problems }\end{array}$ \\
\hline 54. Nielsen et al. & 2007 & Denmark & $325813-18$ years & $\begin{array}{l}\text { SOC, health reported by } \\
\text { teachers and school doctor } \\
\text { (illness symptoms), stress }\end{array}$ & $\begin{array}{l}\text { SOC and stress were associated } \\
\text { with health; girls who report low } \\
\text { SOC and exposure to stress had } \\
\text { more illness symptoms; this } \\
\text { diminished in high SOC girls }\end{array}$ \\
\hline 55. Nio et al. & 2010 & Japan & $\begin{array}{l}12-18 \text { years cardiac } \\
\text { disease }=534 \\
\text { healthy }=406\end{array}$ & $\begin{array}{l}\text { SOC, disease, background } \\
\text { factors }\end{array}$ & $\begin{array}{l}\text { Boys had higher SOC than girls; } \\
\text { sick adolescents higher SOC } \\
\text { than healthy; lower on illness } \\
\text { condition scale higher on SOC }\end{array}$ \\
\hline 56. Neuner et al. & 2011 & Germany & $77014-17$ years longitudinal & $\begin{array}{l}\text { SOC, QoL, heart defects, well- } \\
\text { being, self-esteem }\end{array}$ & $\begin{array}{l}\text { SOC correlated with all QoL, } \\
\text { well-being scales in both times. } \\
\text { The strength of the relationships } \\
\text { differed }\end{array}$ \\
\hline 57. Peled et al. & 2013 & Israel & $28412-18$ years & $\begin{array}{l}\text { Anger, hope, type of } \\
\text { community, community } \\
\text { perceptions based on personal } \\
\text { SOC components }\end{array}$ & $\begin{array}{l}\text { Type of community and } \\
\text { community perceptions } \\
\text { explained anger and hope }\end{array}$ \\
\hline 58. Ristkari et al. & 2009 & Finland & 2314 boys 18 years & $\begin{array}{l}\text { Psychopathology, teachers and } \\
\text { parents reports, self-report, } \\
\text { depression, SOC }\end{array}$ & $\begin{array}{l}\text { Low parental level of education } \\
\text { (at age 8) and non-two } \\
\text { biological parents at home } \\
\text { predicted lower SOC; } \\
\text { internalizing symptoms at } \\
8 \text { years (parents report) and } \\
\text { depression symptoms at } 8 \text { years } \\
\text { (self-report) predicted lower } \\
\text { SOC; comorbidity of } \\
\text { internalizing and conduct } \\
\text { problems had strongest } \\
\text { association with low SOC }\end{array}$ \\
\hline 59. Sagy et al. & 2009 & Israel & $\begin{array}{l}303 \text { Northern- acute stress; } \\
114 \text { South-chronic stress } \\
12-18 \text { years }\end{array}$ & $\begin{array}{l}\text { Anxiety, SPD, SOC, family } \\
\text { SOC, sense of community }\end{array}$ & $\begin{array}{l}\text { Higher anxiety in acute stress } \\
\text { group; higher SPD in chronic } \\
\text { stress group; SOC and family } \\
\text { SOC had better predictive power } \\
\text { in chronic stress group (political } \\
\text { violence) }\end{array}$ \\
\hline $\begin{array}{l}\text { 60. Simonsson } \\
\text { et al. }\end{array}$ & 2008 & Sweden & 399816 and 19 years & Psychosomatic complaints SOC & $\begin{array}{l}\text { Correlations between } \\
\text { psychosomatic symptoms and } \\
\text { SOC. Weak SOC higher } \\
\text { symptoms }\end{array}$ \\
\hline 61. Sollerhed & 2005 & Sweden & $30116-19$ years & $\begin{array}{l}\text { SOC, attitudes to physical } \\
\text { education, exercise in leisure } \\
\text { time, GPA, PE grade, subjective } \\
\text { health, feeling in school, } \\
\text { allocated time to PE }\end{array}$ & $\begin{array}{l}\text { Variables related to attitudes to } \\
\text { PE: strong SOC, high physical } \\
\text { capacity, high leisure time, } \\
\text { physical activity, high grades in } \\
\text { PE, and little time spent } \\
\text { watching TV. } \\
\text { Variables related to strong SOC: } \\
\text { positive attitudes to PE, high } \\
\text { grades in PE, very good } \\
\text { subjective health, and feeling } \\
\text { comfortable in school }\end{array}$ \\
\hline 62. Togari et al. & 2012 & Japan & $\begin{array}{l}1505 \text { adolescents } 989 \text { legal } \\
\text { guardians }\end{array}$ & $\begin{array}{l}\text { Adolescents: SOC, positive life } \\
\text { experience at home. Guardians: } \\
\text { SOC, family relations }\end{array}$ & $\begin{array}{l}\text { Boys: mothers' SOC directly } \\
\text { related to boys' SOC regardless } \\
\text { family relations and } \\
\text { participation in decisions at } \\
\text { homes; Girls: mothers' SOC } \\
\text { indirectly related to girls' SOC } \\
\text { through participation in decision } \\
\text { making at home }\end{array}$ \\
\hline
\end{tabular}


Table 14.1 (continued)

\begin{tabular}{|c|c|c|c|c|c|}
\hline Author & Year & Place & Population & Variables & Results \\
\hline 63. Wang et al. & 2012 & Australia & $\begin{array}{l}11912-20 \text { years with heart } \\
\text { disease }\end{array}$ & $\begin{array}{l}\text { Anxiety, depression, optimism, } \\
\text { social support, SOC }\end{array}$ & $\begin{array}{l}\text { Relatively high anxiety and } \\
\text { depression; optimism, social } \\
\text { support and SOC predicted } \\
\text { anxiety, and depression (in this } \\
\text { order) }\end{array}$ \\
\hline 64. Winding & 2013 & Denmark & $305814-15$ years & $\begin{array}{l}\text { SOC, school performance, } \\
\text { health, vulnerability }\end{array}$ & $\begin{array}{l}\text { Low grades when completing } \\
\text { compulsory school predicted not } \\
\text { having completed a secondary } \\
\text { education by age } 20 / 21 \text {. Low } \\
\text { sense of coherence in childhood } \\
\text { was associated with dropping } \\
\text { out from a vocational education. } \\
\text { Low general health status was } \\
\text { associated with dropping out or } \\
\text { never attaining a secondary } \\
\text { education and overweight was } \\
\text { associated with never attaining a } \\
\text { secondary education }\end{array}$ \\
\hline 65. Woolley & 2006 & USA & $\begin{array}{l}2099 \text { middle and high school } \\
\text { students }\end{array}$ & $\begin{array}{l}\text { School safety, teacher's support, } \\
\text { neighborhood satisfaction and } \\
\text { safety, neighborhood peer } \\
\text { culture, family satisfaction, } \\
\text { family integration, family } \\
\text { support, home academic culture, } \\
\text { problem behavior, school } \\
\text { coherence, academic } \\
\text { performance }\end{array}$ & $\begin{array}{l}\text { Family with cooperative and } \\
\text { supportive interactions such as } \\
\text { work together to solve } \\
\text { problems, provide each other } \\
\text { with loving support, talk about } \\
\text { things youth study in school, and } \\
\text { encourage youth to do well in } \\
\text { school contribute to stronger } \\
\text { school coherence is strongest } \\
\text { among their youth. Also } \\
\text { teacher's support is an important } \\
\text { factor for school coherence }\end{array}$ \\
\hline $\begin{array}{l}\text { 66. Zimprich } \\
\text { et al. }\end{array}$ & 2006 & Switzerland & 1107 7th-9th graders & $\begin{array}{l}\text { Examination of the factorial } \\
\text { structure of SOC } 13\end{array}$ & $\begin{array}{l}\text { For both younger and older } \\
\text { adolescents two factors } \\
\text { emerged: one factor reflected } \\
\text { manageability and } \\
\text { comprehensibility and the other } \\
\text { reflected meaningfulness }\end{array}$ \\
\hline
\end{tabular}

Box 1: Coping Strategies as Mediators of the Relationship Between Sense of Coherence and Stress Reactions: Israeli Adolescents Under Missile Attacks

Orna Braun-Lewensohn, Shifra Sagy, Guy RothAnxiety, Stress \& Coping, 24(3), 327-341: 2011.

Studies on adolescents have indicated that during adolescence SOC may play a protective role similar to that of the mature adult SOC (e.g., Braun-Lewensohn \& Sagy, 2010). However, in several studies conducted during acute stress situations (such as wars, terror, and evacuations), SOC was found weaker effect of explanation of the variance of stress reactions than in chronic stress situations (e.g., Sagy \& BraunLewensohn, 2009). Thus, the present study sought to explore the contribution of SOC in an acute stress situation by trying to find other mediating factors which could explain the outcomes of stress reactions. Employing the interactionist cognitive approach (Lazarus \& Folkman, 1984), we considered two variables that could be significant in mediating the relationship between the personal SOC and stress reactions: cognitive appraisal and coping strategies. The importance of this study is in its being a field research carried out in the midst of the stressful situation of war and severe missile attacks. Although previous studies indicated SOC as a weak factor in explaining stress reactions during acute stressful situations, the present study highlights the possibility that, through the mediating process of coping strategies, SOC could still have high explanatory effect on stress reactions not only in chronic states. The results of this study have drawn attention to the importance of SOC as a resilience factor during an acute stressful situation. 
Other demographic characteristics, apart from age, have significant roles in the determination of the SOC levels. Gender differences were examined, and many studies showed that the SOC scores of boys were higher than the scores of girls (Apres et al., 2013; Dorri, Sheiham, Hardy, \& Watt, 2010; Evans, Marsh, \& Weigel, 2010; Kristensson \& Öhlund, 2005; Moksnes, Rannestad, Byrne, \& Espnes, 2011; Moksnes et al., 2012; Nio, 2010). In addition, socioeconomic status plays an important role in the SOC prediction. Thus, higher levels of parents' education (Feldt, Kokko, Kinnunen, \& Pulkkinen, 2005; Geckova, Tavel, van Dijk, Abel, \& Reijneveld, 2010; Ristkari et al., 2009), higher economic status (Geckova et al., 2010), and living with two parents (Ayo-Yusuf, Reddy, \& Van Den Borne, 2009) have been important indicators of stronger SOC. Lastly, membership in a minority group in different cultures around the world predicted lower SOC than the majority counterparts members (Braun-Lewensohn, 2014; Braun-Lewensohn \& Sagy, 2011a, 2011b; Glanz, Gertraud, \& Carlin, 2005).

\section{SOC, Health, Mental Health, and Psychosocial Behavior}

Examining the various studies, we found that the relations of health, mental health, and psychosocial behaviors with SOC were explored. More specifically, researchers investigated the SOC as a predictor of health outcomes, mental health, and diverse health promoting behaviors during adolescence.

Several studies examined the relations between the SOC and general health. They reported that higher SOC has been related to a better perceived health while lower SOC was related to greater amounts of medication usage. Moreover, SOC was negatively related to reported health problems (Blom, Serlachius, Larsson, Theorell, \& Ingvar, 2010; García-Moya et al., 2013; Geckova et al., 2010; Honkinen, Suominen, Välimaa, Helenius, \& Rautava, 2005; Koushede \& Holstein, 2009; Mattila et al., 2011; Modin, Östberg, Toivanen, \& Sundell, 2011; Myrin \& Lagerström, 2006; Moksnes et al., 2011).

Other examinations related to health focused on groups with specific health problems. For example, surprisingly, adolescents with heart problems were found to have higher SOC compared to healthy adolescents. These results were explained by the fact that youngsters with such chronic disease have learned to cope with their problem which increased their manageability, besides having high existential implications that increases their meaningfulness. Moreover, a supportive home environment experienced by these adolescents emphasize specific life events as being more comprehensible, manageable, and meaningful hence, nurtured feelings of SOC (Luyckx, Missotten, Goossens, \& Moons, 2012). More expected results were found for adolescents with epilepsy where decreased SOC was found in the long run, reflecting the experience of losing control during seizures and difficulty in assessing when to expect the next seizure. Following this line, those adolescents with no seizures had higher SOC (Gauffin, Landtblom, \& Räty, 2010).

Mental health has been the focus of numerous studies that examined diverse outcomes. Stress-related outcomes such as anxiety, anger, depression, psychological distress, and other emotional and internalizing or externalizing problems were examined in the context of political violence (BraunLewensohn \& Sagy, 2011a, 2011b, 2010; Braun-Lewensohn \& Sagy, 2010; Sagy \& Braun-Lewensohn, 2009) and with regard to challenging and extreme life events such as child abuse (Gustafsson et al., 2010) or juvenile delinquency (Koposov et al., 2003). However, adolescents were also examined during regular daily life with 'normal' life stressors, such as academic, school, or peer pressure as well as family conflicts (Moksnes et al., 2012; Moksnes, Espnes, \& Haugan, 2013; Nielsen \& Hansson, 2007; Ristkari et al., 2009; Simonsson, Nilsson, Leppert, \& Diwan, 2008). All these studies confirm that the SOC can be considered a resilient factor. It can be concluded that a strong SOC predicts reduced stress and decreased internalizing or/and externalizing problems.

Moreover, examining the relationships of SOC with psychosocial behaviors even strengthens the consideration of SOC as a resilient factor. Accordingly, results of various studies showed that adolescents with higher SOC reported a more healthy life style, a better quality of life, and wellbeing (Honkinen et al., 2009; Neuner et al., 2011). The healthy lifestyle related on one hand to physical activities and exercises (Bronikowski, 2010) and on the other hand to smoking habits, alcohol abuse (García-Moya et al., 2013, 2013a; Myrin \& Lagerström, 2006; Nielsen \& Hansson, 2007), and eating habits (Myrin \& Lagerström, 2006). Similarly, the relations between SOC and oral behavior were reported. Higher SOC was linked to lower gingivitis, more willingness to change tooth brush habits, and especially increased tooth brushing (e.g., Ayo-Yusuf, Reddy, \& Van Den Borne, 2008, 2009; Dorri et al., 2010).

\section{Ecological Contexts: Family, School, Peers, and Community}

Ecological contexts (Bronfenbrenner, 1977, 1979; Bronfenbrenner \& Morris, 2006) extend the consideration from a focus on personal levels to awareness and sensitization to contextual characteristics and systemic consideration such as the families, schools, and communities. Several, family-related factors were examined in relation to SOC. For example, open family communication (García-Moya 
et al., 2013; Marsh et al., 2007) focused parenting style (García-Moya et al., 2013) and parents' knowledge regarding their children activities (García-Moya et al., 2013) were considered positive contributors to the development of a strong SOC. In addition, child-centered parenting during adolescence (examined within a longitudinal paradigm) predicted a stronger SOC at adulthood (Feldt et al., 2005).

In addition to examining family contexts and factors which could enhance or reduce personal SOC, few studies also related to family sense of coherence as another source to rely on when facing difficulties and/or stressful situations. Likewise, the personal SOC it was found that also family SOC is a resilient factor, and adolescents with stronger family SOC reported reduced stress (Sagy \& BraunLewensohn, 2009; Sagy \& Dotan, 2001).

Another important ecological system is the school. While the family dimension produced mainly studies that pinpointed attention at the contribution of family characteristics to the development of SOC, studies of schools focused attention on outcomes, examining the adolescents' achievement and adjustment, and their relationship to SOC as a mediation factor. Within the educational systems, a stronger SOC predicted high grades, enhanced academic motivation, and success. A lower stress levels were also reported as related to stronger SOC (Honkinen et al., 2005; Kristensson \& Öhlund, 2005; Lackaye \& Margalit, 2006). Moreover, stronger SOC was linked to social competence (Mattila et al., 2011; Moksnes et al., 2011).

The school system provides a unique opportunity to look at special populations with regard to SOC. Adolescents with learning disabilities are an additional example to the importance of the SOC (Idan \& Margalit, 2014; Lackaye \& Margalit, 2006). These youngsters are identified by their chronic academic challenges emerging from neurodevelopmental difficulties. Their difficulties at school systems remain a continuous source for increased stress, endless day-to-day struggling with age-appropriate academic roles, and with social and emotional consequences. Indeed, their sources of stress are not dramatic, but their chronic impact are expressed in lower SOC. Studies placed the SOC as a mediator of hopeful thinking, predicting adjustment and effort investment in school. The adolescents' systems, such as families, schools and communities, may further clarify the important role of the SOC and the factors that predict its development.

The focus on peer relations and community atmosphere produced studies which explored these factors as predictors to SOC development. An additional group of studies explored SOC as a collective construct contributing to the mental health of adolescents. Exploring SOC as dependent variable, it seems that social support (Marsh et al., 2007) neighborhood or community cohesion (García-Moya et al.,
2013; Marsh et al., 2007; Peled, Sagy, \& Braun-Lewensohn, 2013) and success in school (García-Moya et al., 2013) are all constructive in the development of strong SOC.

In a different vein, and as an experience to expand SOC from personal to collective level an adjusted instrument, sense of community coherence, that includes Antonovsky's components of personal SOC-comprehensibility, manageability, and meaningfulness (Braun-Lewensohn, 2014; Braun-Lewensohn \& Sagy, 2011b; Peled et al., 2013) was developed. Comprehensibility refers to the sense of predictability, safety, and security felt by members of the community and the extent to which that community is understandable. A community's manageability expresses its ability to assist its members, via treatment providers and group programs, among others, in times of crisis and distress. Lastly, the higher the level of meaningfulness among the members of a community, the more able they are to express themselves and the greater the likelihood that they will feel satisfied with and challenged and interested by what the community has to offer them (Braun-Lewensohn \& Sagy, 2011b; Sagy \& Dotan, 2001). Recent studies showed that indeed sense of community coherence is another source of support to effective coping during adolescence when facing acute or chronic types of stress especially among collectivist cultures (Braun-Lewensohn, 2014; BraunLewensohn \& Sagy, 2011a, 2011b; Peled et al., 2013).

\section{Conclusions, Implications, and Future Research Directions}

This chapter focused on sense of coherence and salutogenesis during the developmental period of adolescence. As noted earlier in this chapter, the uniqueness of this developmental stage is the many challenges and changes which individuals are going through during these years. While in many ways the adolescents appear to function similar to adults, there are numerous cognitive, biological, and behavioral processes which are being formed and shaped during these years in the paths to maturity and normative adulthood.

A recent review on salutogenesis and the concept of SOC examined the influence of different factors such as gender and age as well as different developmental contexts (family, school, peers, and neighborhood) on the development of SOC (Rivera et al., 2013). In our current review, we extended conceptualization and research results regarding SOC during this important developmental period of adolescence within a different orientation. Mainly we addressed the way in which the SOC questionnaire was adopted to fit adolescent populations, as well as, the clarifying ways how SOC is linked to different health, mental health, and psychosocial behavior in different ecological contexts. We 
conclude that the review of studies from around the world in the last decade demonstrated that personal and systemic (i.e., family and community) SOC are meaningful resources for effective coping with a wide variety of stressful situations. The survey of the studies shows that the SOC may be considered as a protective factor for adolescents in different cultures. During adolescence, the SOC may contribute to moderating and mediating stress experiences and may also play a protective role similar to that of the mature adult SOC.

The educational and community implications of the current consideration of the SOC as a critical resource, calls for the sensitizing educators and community workers to the importance of the salutogenic construct. Future empowering programs should be guided by this construct, leading to the development of prevention/inoculation to stress planning as well as programs promoting positive psychosocial and healthy behaviors and academic success.

Our review raises several directions for future research in the field of salutogenesis and sense of coherence during adolescence. In spite of the many studies that have been conducted and published in the last decades and the important developments in this field, there are still some venues that have been neglected. First, the role of family coherence as a protective factor for health and mental health as well as relationships with success in school and other psychosocial behaviors should be further explored. Moreover, non-Western cultural groups were less studied in this context. When studied, some questions regarding the universality of the concept of SOC were raised (Braun-Lewensohn \& Sagy, 2011a, 2011b). Thus, it seems important to further examine this issue. We should focus on the meanings of sense of coherence in such cultures as well as the understanding of the questionnaire and the implication of SOC in such societies.

Box 2: Coping Resources and Stress Reactions Among Three Cultural Groups One Year After a Natural Disaster

Orna Braun-Lewensohn Clinical Social Work, DOI:10.1007/s10615-013-0463-0: 2014

A year after a huge bush fire, adolescents from three cultures-Jews, Muslims, and Druze-located in the Carmel district (the area of the fire) were asked to report their personal and community sense of coherence (SOC) as well as their stress reactions of anxiety, anger, and psychological distress. We wanted to examine Antonovsky's conviction (1987) that sense of coherence (SOC) is a cross-cultural concept by comparing adolescents belonging to the majority group, an individualistic culture (Jews), to members of two minority groups which are collectivistic cultures (Muslim and Druze), in terms of personal and community SOC as well as on stress reactions. We also wanted to determine whether the coping resources explained similarly or differently the stress reactions which were examined. Results show that although levels of personal SOC varied significantly across the groups, with the majority group having the strongest sense of coherence, personal SOC had a strong protective effect against stress reactions in all groups. Community SOC, in turn, had a protective effect only for members of collectivist culture. We can cautiously conclude, therefore, that it may be possible for different cultures to have their own, culturally relevant translations for SOC so that it becomes a meaningful protective factor when confronting stressful situations.

Open Access This chapter is distributed under the terms of the Creative Commons Attribution-Noncommercial 2.5 License (http:// creativecommons.org/licenses/by-nc/2.5/) which permits any noncommercial use, distribution, and reproduction in any medium, provided the original author(s) and source are credited.

The images or other third party material in this chapter are included in the work's Creative Commons license, unless indicated otherwise in the credit line; if such material is not included in the work's Creative Commons license and the respective action is not permitted by statutory regulation, users will need to obtain permission from the license holder to duplicate, adapt or reproduce the material.

\section{References}

Aldwin, C. M. (1994). Stress, coping, and development: An integrative perspective. New York: Guilford Press.

Antonovsky, A. (1987). Unraveling the mystery of health: How people manage stress and stay well. San Francisco: Jossey-Bass.

Ayo-Yusuf, O. A., Reddy, P. S., \& Van Den Borne, B. W. (2008). Adolescents' sense of coherence and smoking as longitudinal predictors of self-reported gingivitis. Journal of Clinical Periodontology, 35(11), 931-937.

Antonovsky, A. (1993). The structure and properties of the sense of coherence scale. Social science \& medicine, 36(6), 725-733.

Antonovsky, H., \& Sagy, S. (1986). The development of a sense of coherence and its impact on responses to stress situations. Journal of Social Psychology, 126(2), 213-226.

Apers, S., Moons, P., Goossens, E., Luyckx, K., Gewillig, M., Bogaerts, K., et al. (2013). Sense of coherence and perceived physical health explain the better quality of life in adolescents with congenital heart disease. European Journal of Cardiovascular Nursing, 12(5), 475483.

Ayo-Yusuf, O. A., Reddy, P. S., \& Van Den Borne, B. W. (2009). Longitudinal association of adolescents' sense of coherence with tooth-brushing using an integrated behaviour change model. Community Dentistry and Oral Epidemiology, 37(1), 68-77. 
Blakemore, S. -J., \& Mills, K. L. (2014). Is adolescence a sensitive period for sociocultural processing? Annual Review of Psychology, 65(1), 187-207. doi:10.1146/annurev-psych-010213-115202

Blom, E. C. H., Serlachius, E., Larsson, J. O., Theorell, T., \& Ingvar, M. (2010). Research low sense of coherence (SOC) is a mirror of general anxiety and persistent depressive symptoms in adolescent girls-a cross-sectional study of a clinical and a non-clinical cohort. Health and Quality of Life Outcomes, 8, 58.

Braun-Lewensohn, O. (2014). Coping resources and stress reactions among three cultural groups one year after a natural disaster. Clinical Social Work Journal, 42, 366-374.

Braun-Lewensohn, O., \& Sagy, S. (2010). Sense of coherence, hope and values among adolescents under missile attacks: A longitudinal study. International Journal of Children's Spirituality, 15(3), 247-260.

Braun-Lewensohn, O., Sagy, S., Sabato, H. \& Galili, R. (2013). Sense of coherence and sense of community as coping resources of religious adolescents before and after the disengagement from the Gaza Strip. Israeli Journal of Psychiatry and Related Sciences, 50(2), 110-117.

Braun-Lewensohn, O., \& Sagy, S. (2011a). Salutogenesis and culture: Personal and community sense of coherence among adolescents belonging to three different cultural groups. International Review of Psychiatry, 23(6), 533-541.

Braun-Lewensohn, O., \& Sagy, S. (2011b). Coping resources as explanatory factors of stress reactions during missile attacks: Comparing Jewish and Arab adolescents in Israel. Community Mental Health Journal, 47(3), 300-310.

Bronfenbrenner, U. (1977). Toward an experimental ecology of human development. American Psychologist, 32(7), 513.

Bronfenbrenner, U. (1979). Contexts of child rearing: Problems and prospects. American Psychologist, 34(10), 844.

Bronfenbrenner, U., \& Morris, P. A. (2006). The bioecological model of human development. In R. M. L. W. Damon (Ed.), Handbook of child psychology: Vol. 1, Theoretical models of human development (6th ed., pp. 793-828). Hoboken, NJ: Wiley.

Bronikowski, M. (2010). Is sense of coherence needed to keep youth physically active? MEDICINA DELLO SPORT, 63(4), 465-483.

Buddeberg-Fischer, B., Klaghofer, R., \& Schnyder, U. (2001). Sense of coherence in adolescents. Social and Preventive Medicine, 46(6), 404-410.

Casey, B. J., \& Caudle, K. (2013). The teenage brain: Self control. Current directions in psychological science, 22(2), 82-87.

Damon, W. (2004). What is positive youth development. Annals of the American Academy of Political and Social Science, 591, 13-30.

Dorri, M., Sheiham, A., Hardy, R., \& Watt, R. (2010). The relationship between Sense of Coherence and toothbrushing behaviours in Iranian adolescents in Mashhad. Journal of Clinical Periodontology, 37(1), 46-52.

Eriksson, M. (2007). Unravelling the mystery of salutogenesis: the evidence base of the salutogenic research as measured by Antonovsky's Sense of Coherence Scale. Turku, Fenland: Folkhälsan Research Centre.

Evans, W. P., Marsh, S. C., \& Weigel, D. J. (2010). Promoting adolescent sense of coherence: Testing models of risk, protection, and resiliency. Journal of Community \& Applied Social Psychology, 20 (1), 30-43.

Feldt, T., Kokko, K., Kinnunen, U., \& Pulkkinen, L. (2005). The role of family background, school success, and career orientation in the development of sense of coherence. European Psychologist, 10(4), 298-308.

García-Moya, I., Jiménez-Iglesias, A., \& Moreno, C. (2013). Sense of coherence and substance use in Spanish adolescents. Does the effect of SOC depend on patterns of substance use in their peer group? Adicciones, 25(2), 109.
García-Moya, I., Moreno, C., \& Braun-Lewensohn, O. (2013). Neighbourhood perceptions and sense of coherence in adolescence. The Journal of Primary Prevention, 34(5), 371-379.

García-Moya, I., Moreno, C., \& Jiménez-Iglesias, A. (2013). Understanding the joint effects of family and other developmental contexts on the sense of coherence (SOC): A person-focused analysis using the classification tree. Journal of Adolescence, 36(5), 913-923.

García-Moya, I., Rivera, F., \& Moreno, C. (2013). School context and health in adolescence: The role of sense of coherence. Scandinavian Journal of Psychology, 54(3), 243-249.

Garnefski, N., Kraaij, V., \& Spinhoven, P. (2001). Negative life events, cognitive emotion regulation and emotional problems. Personality and Individual Differences, 30(8), 1311-1327.

Gauffin, H., Landtblom, A. M., \& Räty, L. (2010). Self-esteem and sense of coherence in young people with uncomplicated epilepsy: A 5-year follow-up. Epilepsy \& Behavior, 17(4), 520-524.

Geckova, A. M., Tavel, P., van Dijk, J., Abel, T., \& Reijneveld, S. (2010). Factors associated with educational aspirations among adolescents: Cues to counteract socioeconomic differences? BMC Public Health, 10(1), 154.

Glanz, K., Gertraud, M., \& Carlin, L. (2005). Ethnicity, sense of coherence, and tobacco use among adolescents. Annals of Behavioral Medicine, 29(3), 192-199.

Gustafsson, P. E., Nelson, N., \& Gustafsson, P. A. (2010). Diurnal cortisol levels, psychiatric symptoms and sense of coherence in abused adolescents. Nordic Journal of Psychiatry, 64(1), 27-31.

Hagquist, C., \& Andrich, D. (2004). Is the sense of coherenceinstrument applicable on adolescents? A latent trait analysis using Rasch-modelling. Personality and Individual Differences, 36(4), 955-968.

Honkinen, P. L., Aromaa, M., Suominen, S., Rautava, P., Sourander, A., Helenius, H., et al. (2009). Early childhood psychological problems predict a poor sense of coherence in adolescents a 15-year follow-up study. Journal of Health Psychology, 14(4), 587-600.

Honkinen, P. L., Suominen, S., Helenius, H., Aromaa, M., Rautava, P., Sourander, A., et al. (2008). Stability of the sense of coherence in adolescence. International Journal of Adolescent Medicine and Health, 20, 85-91.

Honkinen, P. L. K., Suominen, S. B., Välimaa, R. S., Helenius, H. Y., \& Rautava, P. T. (2005). Factors associated with perceived health among 12-year-old school children. Relevance of physical exercise and sense of coherence. Scandinavian Journal of Public Health, 33 (1), 35-41.

Idan, O., \& Margalit, M. (2014). Socioemotional self-perceptions, family climate, and hopeful thinking among students with learning disabilities and typically achieving students from the same classes. Journal of Learning Disabilities, 47(2), 136-152.

Koposov, R. A., Ruchkin, V. V., \& Eisemann, M. (2003). Sense of coherence: A mediator between violence exposure and psychopathology in Russian juvenile delinquents. The Journal of Nervous and Mental Disease, 191(10), 638-644.

Koushede, V., \& Holstein, B. E. (2009). Sense of coherence and medicine use for headache among adolescents. Journal of Adolescent Health, 45(2), 149-155.

Kristensson, P., \& Öhlund, L. S. (2005). Swedish upper secondary school pupils' sense of coherence, coping resources and aggressiveness in relation to educational track and performance. Scandinavian Journal of Caring Sciences, 19(1), 77-84.

Kröninger-Jungaberle, H., \& Grevenstein, D. (2013). Development of salutogenetic factors in mental health Antonovsky's sense of coherence and Bandura's self-efficacy related to Derogatis' symptom check list (SCL-90-R). Health and Quality of Life Outcomes, $11(1), 1$. 
Lackaye, T. D., \& Margalit, M. (2006). Comparisons of achievement, effort, and self-perceptions among students with learning disabilities and their peers from different achievement groups. Journal of Learning Disabilities, 39(5), 432-446.

Lazarus, R. S., \& Folkman, S. (1984). Stress, appraisal, and coping. New York: Springer.

Lerner, R. M., \& Benson, P. I. (2003). Developmental assets and assetbuilding communities: Implications for research, policy, and practice. New York: Kluwer Academic/Plenum.

Levi, U., Einav, M., Ziv, O., Raskind, I., \& Margalit, M. (2014). Academic expectation and actual achievement: The roles of hope and effort. European Journal of Psychology of Education, 29(3), 367-386.

Luyckx, K., Missotten, L., Goossens, E., \& Moons, P. (2012). Individual and contextual determinants of quality of life in adolescents with congenital heart disease. Journal of Adolescent Health, 51(2), $122-128$.

Marsh, S. C., Clinkinbeard, S. S., Thomas, R. M., \& Evans, W. P. (2007). Risk and protective factors predictive of sense of coherence during adolescence. Journal of Health Psychology, 12(2), 281-284.

Mattila, M. L., Rautava, P., Honkinen, P. L., Ojanlatva, A., Jaakkola, S., \& Aromaa, M., et al. (2011). Sense of coherence and health behaviour in adolescence. Acta Paediatrica, 100(12), 1590-1595.

Modin, B., Östberg, V., Toivanen, S., \& Sundell, K. (2011). Psychosocial working conditions, school sense of coherence and subjective health complaints. A multilevel analysis of ninth grade pupils in the Stockholm area. Journal of Adolescence, 34(1), 129-139.

Moksnes, U. K., Espnes, G. A., \& Haugan, G. (2013). Stress, sense of coherence, and emotional symptoms in adolescents. Psychology \& Health, 29(1), 32-49.

Moksnes, U. K., Espnes, G. A., \& Lillefjell, M. (2012). Sense of coherence and emotional health in adolescents. Journal of Adolescence, 35(2), 433-441.

Moksnes, U. K., Rannestad, T., Byrne, D. G., \& Espnes, G. A. (2011). The association between stress, sense of coherence and subjective health complaints in adolescents: Sense of coherence as a potential moderator. Stress and Health, 27(3), e157-e165.

Myrin, B., \& Lagerström, M. (2006). Health behaviour and sense of coherence among pupils aged 14-15. Scandinavian Journal of Caring Sciences, 20(3), 339-346.

Neuner, B., Busch, M. A., Singer, S., Moons, P., Wellmann, J., Bauer, U., et al. (2011). Sense of coherence as a predictor of quality of life in adolescents with congenital heart defects: A register-based 1-year follow-up study. Journal of Developmental \& Behavioral Pediatrics, 32(4), 316-327.

Nielsen, A. M., \& Hansson, K. (2007). Associations between adolescents' health, stress and sense of coherence. Stress and Health, 23(5), 331-341.

Nio, K. (2010). Sense of coherence in adolescents with congenital cardiac disease. Cardiology in the Young, 20(5), 538.

Peled, D., Sagy, S., \& Braun-Lewensohn, O. (2013). Community perception as coping resource among adolescents living under rockets fire: A salutogenic approach. Journal of Community Positive Practices, 4, 681-702.

Ristkari, T., Sourander, A., Rønning, J. A., Piha, J., Kumpulainen, K., Tamminen, T., et al. (2009). Childhood psychopathology and sense of coherence at age 18: findings from the Finnish from a boy to a man study. Social Psychiatry and Psychiatric Epidemiology, 44 (12), 1097-1105.

Rivera, F., García-Moya, I., Moreno, C., \& Ramos, P. (2013). Developmental contexts and sense of coherence in adolescence: A systematic review. Journal of Health Psychology, 18(6), 800-812. doi: $10.1177 / 1359105312455077$.

Romeo, R. D. (2013). The teenage brain: The stress response and the adolescent brain. Current Directions in Psychological Science, 22, 140-145.

Sagy, S., \& Braun-Lewensohn, O. (2009). Adolescents under rocket fire: When are coping resources significant in reducing emotional distress? Global Health Promotion, 16(4), 5-15.

Sagy, S., \& Dotan, N. (2001). Coping resources of maltreated children in the family: A salutogenic approach. Child Abuse \& Neglect, 25 (11), 1463-1480.

Simonsson, B., Nilsson, K. W., Leppert, J., \& Diwan, V. K. (2008). Psychosomatic complaints and sense of coherence among adolescents in a county in Sweden: A cross-sectional school survey. BioPsychoSocial Medicine, 2(4), 8.

Somerville, L. H., Hare, T., \& Casey, B. J. (2011). Frontostriatal maturation predicts cognitive control failure to appetitive cues in adolescents. Journal of Cognitive Neuroscience, 23, 2123-2134.

Spear, L. (2013). The teenage brain: Adolescents and alcohol. Current Directions in Psychological Science, 22, 152-157.

Tottenham, N., Hare, T. A., \& Casey, B. J. (2011). Behavioral assessment of emotion discrimination, emotion regulation, and cognitive control in childhood, adolescence, and adulthood. Frontiers in Psychology, 2, 39. doi:10.3389/fpsyg.2011.00039. 